\title{
Part II: Beneficial Effects of the Peroxynitrite Decomposition Catalyst FP15 in Murine Models of Arthritis and Colitis
}

\author{
Jon G. Mabley, ${ }^{1}$ Lucas Liaudet, ${ }^{2,3}$ Pál Pacher, ${ }^{1}$ Garry J. Southan, ${ }^{1}$ Andrew L. Salzman, ${ }^{1}$ \\ and Csaba Szabó ${ }^{1,2}$ \\ ${ }^{1}$ Inotek Pharmaceuticals Corporation, Cummings Center, Beverly, Massachusetts, USA \\ ${ }^{2}$ Department of Surgery, New Jersey Medical School, University of Medicine and Dentistry, New Jersey, \\ Newark, New Jersey, USA \\ ${ }^{3}$ Critical Care Division, Department of Internal Medicine, University Hospital, Lausanne, Switzerland \\ Accepted August 21, 2002. Contributed by C. Szabó
}

\begin{abstract}
Background: Peroxynitrite is a reactive oxidant species produced from nitric oxide and superoxide, which has been indirectly implicated in the pathogenesis of many inflammatory conditions including arthritis and colitis. Here, using a novel peroxynitrite decomposition catalyst, FP15, we directly investigate the role of peroxynitrite in the pathogenesis of arthritis and colitis in rodent models. Methods: Arthritis was induced in mice by intradermal collagen injection; incidence and severity of arthritis was monitored using a macroscopic scoring system. At the end of the experiment paws were taken for determination of neutrophil infiltration (myeloperoxidase [MPO] activity), oxidative stress (malondialdehyde [MDA] level), and cytokine/chemokine levels. Colitis was induced in mice by $5 \%$ dextran sodium sulfate (DSS) in their drinking water. Colitis symptoms were assessed 10 days later, the
\end{abstract}

parameters determined included body weight, rectal bleeding, colon length, colonic MPO and MDA levels, and colon histologic damage.

Results: Treatment with FP15 significantly reduced the inflammation and oxidative stress in arthritis and colitis. FP15 reduced both the incidence and severity of arthritis in mice and this was associated with reduced paw MPO and MDA levels. Similarly, in colitis, FP15 reduced colon damage, and this was associated with reduced colon neutrophil infiltration and oxidative stress.

Conclusions: The protective effect of FP15 suggests that peroxynitrite plays a significant pathogenetic role in arthritis and colitis in the currently employed rodent models. Further work is needed to determine whether neutralization of peroxynitrite also represents a promising strategy to treat human inflammatory diseases such as arthritis and colitis.

\section{Introduction}

There is substantial evidence for the involvement of oxidative stress in the pathogenesis of both colitis and arthritis. Tissue damage in arthritis and colitis is associated with profound alterations in the biosynthesis of the labile free radical nitric oxide from L-arginine. The production of nitric oxide represents a final common pathway that follows the production of the pro-inflammatory cytokines tumor necrosis factor $\alpha$ (TNF- $\alpha)$, interleukin $1 \beta(\mathrm{IL}-1 \beta)$, and interferon $\gamma($ IFN- $\gamma)$. The inducible isoform of nitric oxide synthase (iNOS), which generates nitric oxide (NO) for longer periods of time (several hours to days) and at rates several orders of magnitude greater than the constitutive isoform is not present in the joints or the colon to any significant extent under resting conditions, but is newly synthesized in response to pro-inflammatory cytokines. Expression of iNOS has been observed in experimental models of colitis (1) and specific inhibitors of iNOS have

Address correspondence and reprint requests to: Csaba Szabó, Inotek Pharmaceuticals, Suite 419E, 100 Cummings Center, Beverly, MA 01915. Phone: (978) 232-9660; fax: (978) 232 8975; e-mail: szabocsaba@aol.com. proved effective in ameliorating symptoms of colonic inflammation (2). Colonic inflammation has also been reduced by treatment with a superoxide dismutase (SOD) mimetic (3). In human subjects, urinary excretion of nitrite is well correlated with other markers of colonic inflammation such as C-reactive protein and microalbuminuria (4) and more recently, using a chemiluminescence technique, NO production directly from the colon mucosa has been measured and shown to be increased in patients with colitis (5).

There is now solid evidence that in arthritis both chondrocytes and macrophages express iNOS, with the resulting production of large, cytotoxic amounts of NO within inflamed joints (6). NO can be directly cytotoxic but can also mediate joint destruction by activating metalloproteinase enzymes (collagenase and stromelysin) in the cartilage. In addition, inhibitors of NOS $(7,8)$ as well as SOD mimetics $(9)$ protect against the development of arthritis in rodents. Human cartilage explants produce large amounts of $\mathrm{NO}$ in response to immunostimulation (10); in patients with rheumatoid arthritis, there is increased urinary excretion of nitrate, the stable breakdown product of NO (11). 
Simultaneous generation of NO and superoxide favors the production of a toxic reaction product peroxynitrite $\left(\mathrm{ONOO}^{-}\right)(12)$. $\mathrm{ONOO}^{-}$is more cytotoxic than NO or superoxide in a variety of experimental systems. For instance, in vitro studies demonstrate that NO itself has extremely limited effects on aconitase activity, whereas $\mathrm{ONOO}^{-}$is a potent inhibitor of aconitase under the same experimental conditions $(13,14)$. Similarly, $\mathrm{ONOO}^{-}$, not $\mathrm{NO}$, is the potent generator of DNA strand breaks (15). $\mathrm{ONOO}^{-}$is highly reactive and oxidizes sulfhydryl groups and thioethers, as well as nitrosates hydroxylated aromatic compounds, including tyrosine (16). Tyrosine nitrosation may lead to dysfunction of these proteins (16). $\mathrm{ONOO}^{-}$directly inactivates certain mitochondrial enzymes that are important for the energetic balance of the cell.

In both colitis and arthritis, nitric oxide synthase inhibitors and superoxide dismutase mimetics were found to be protective $(3,9)$. In addition immunohistochemical techniques have demonstrated increased nitrotyrosine in both colitis $(1,2,17)$ and arthritis $(8,18,19)$, suggesting the formation of peroxynitrite plays a pathophysiologic role in these diseases. Indeed, intracolonic administration of peroxynitrite leads to development of colitis (20). The proposal that $\mathrm{ONOO}^{-}$is a major cytotoxic mediator in inflammation would unify previous data demonstrating the protective role of both NO- and superoxide-neutralizing strategies in these models. We have utilized a novel $\mathrm{ONOO}^{-}$ decomposition catalyst, FP15 (21), to directly determine the contribution of peroxynitrite to the pathogenesis of the disease in murine models of colitis and arthritis.

\section{Methods}

Animal Models

All animal experiments were carried out in accordance with the guidelines published by the NIH in Principles of Laboratory Animal Care (NIH publication no. 85-23, revised 1985) and with the approval of the local Institutional Animal Care and Use committee. The animals are housed in rooms at a controlled temperature and light/dark cycle for $48 \mathrm{hr}$ prior to starting experimental protocols.

\section{Induction of Arthritis and Treatment}

Male DBA/IJ mice, 6 weeks of age, were injected intradermally on day 1 with $0.1 \mathrm{ml}$ of an emulsion of bovine type II collagen plus complete Freund's adjuvant. A second injection was administered 3 weeks later (18). Treatment with FP15 (3 or $10 \mathrm{mg} / \mathrm{kg} / \mathrm{d}$ BID) orally was started on the day of the second collagen/CFA injection or on day 7 after the second collagen injection and continued throughout the study, which was terminated 30 days after the second injection. The mice were evaluated daily for arthritis by using a macroscopic scoring system ranging from $0-4(0=$ no signs of arthritis, $1=$ swelling or redness of the paw or one digit, $2=$ two joints involved, $3=$ more than two joints involved, $4=$ severe arthritis of the entire paw). The arthritic index for each mouse was calculated by adding the four scores of the individual paws. Severity indices were calculated for the whole groups of mice (vehicle- or FP15-treated), with no animal being excluded from the calculations, as well as the percentage of the treatment group exhibiting signs of arthritis. When the study was terminated, paws were removed from all animals in each treatment group and randomly assigned for MPO, MDA, or chemokine/cytokine measurements.

\section{Induction of Colitis and Treatment}

Male BALB/c mice, 8 weeks of age, weighing 20-23 g were utilized for these studies. The mice were fed $5 \%$ dextran sulfate sodium (DSS), molecular weight 30-40 kDa dissolved in distilled water ad libitum throughout the experiment. FP15 (1-10 mg/kg/d) was administered orally by gavage twice a day. The vehicle for FP15 was water; control mice were given the same volume of vehicle. Intake of the DSS solution was monitored throughout the experiments was found to be unchanged between experimental groups (data not shown).

\section{Evaluation of Colitis Severity and Drug Effects}

The parameters recorded in the experiments were body weight, colon length, mortality, and bleeding from the rectum as determined by ocular inspection. Mice were weighed on days 1 and 10 with the subsequent colitis-induced weight loss expressed as a percentage of original weight. The mice were killed by cervical dislocation and the colon resected between the ileocecal junction and the proximal rectum close to its passage under the pelvisternum. The colon was placed on a nonabsorbent surface and measured with a ruler. Colonic biopsies were taken for histologic and biochemical analysis. One biopsy was fixed in $15 \%$ formaldehyde, embedded in paraffin, and sectioned $(4-\mu \mathrm{m}$ slices). The sections were then stained with H\&E, viewed by a blinded investigator, and scored for inflammation severity $(0=$ none, $1=$ mild, $2=$ moderate, and $3=$ severe $)$ and extent $(0=$ none, $1=$ mucosal, $2=$ mucosal and submucosal, and $3=$ transmural $)$ as well as crypt damage $(0=$ none, 1 = basal third, 2 = basal two-thirds, $3=$ crypts lost epithelium present, and $4=$ crypts and surface epithelium lost) with representative sections being shown here.

\section{Myeloperoxidase Activity}

Mouse paws or colon biopsies were homogenized (50 $\mathrm{mg} / \mathrm{ml}$ ) in $0.5 \%$ hexadecyltrimethylammonium bromide in $10 \mathrm{mM}$ 3-N-morpholinopropanesulfonic acid (MOPS) and centrifuged at 15,000 $\mathrm{g}$ for $40 \mathrm{~min}$. The suspension was then sonicated three times for $30 \mathrm{sec}-$ onds. An aliquot of supernatant $(20 \mu \mathrm{l})$ was mixed with a solution of $1.6 \mathrm{mM}$ tetra-methyl-benzidine and 
$1 \mathrm{mM}$ hydrogen peroxide. Activity was measured spectrophotometrically as the change in absorbance at $650 \mathrm{~nm}$ at $37^{\circ} \mathrm{C}$, using a Spectramax microplate reader (Molecular Devices, Sunnyvale, CA, USA) (22). Results are expressed as milliunits of myeloperoxidase (MPO) activity per milligram of protein, which were determined with the Bradford assay (23).

\section{Malondialdehyde Assay}

Malondialdehyde formation was utilized to quantify the lipid peroxidation in the mouse paws or colon and measured as thiobarbituric acid-reactive material. Tissues were homogenized $(100 \mathrm{mg} / \mathrm{ml})$ in $1.15 \% \mathrm{KCl}$ buffer. Two hundred microliters of the homogenates were then added to a reaction mixture consisting of $1.5 \mathrm{ml} 0.8 \%$ thiobarbituric acid, $200 \mu \mathrm{l}$ $8.1 \%$ sodium dodecyl sulfate, $1.5 \mathrm{ml} 20 \%$ acetic acid (pH 3.5), and $600 \mu \mathrm{l}$ distilled $\mathrm{H}_{2} \mathrm{O}$. The mixture was then heated at $90{ }^{\circ} \mathrm{C}$ for $45 \mathrm{~min}$. After cooling to room temperature, the samples were cleared by centrifugation $(10,000 \mathrm{~g}$ for $10 \mathrm{~min})$ and their absorbance measured at $532 \mathrm{~nm}$, using 1,1,3,3-tetramethoxypropane as an external standard (22). The level of lipid peroxides was expressed as nmol of malondialdehyde assay (MDA) per milligram of protein (Bradford assay).
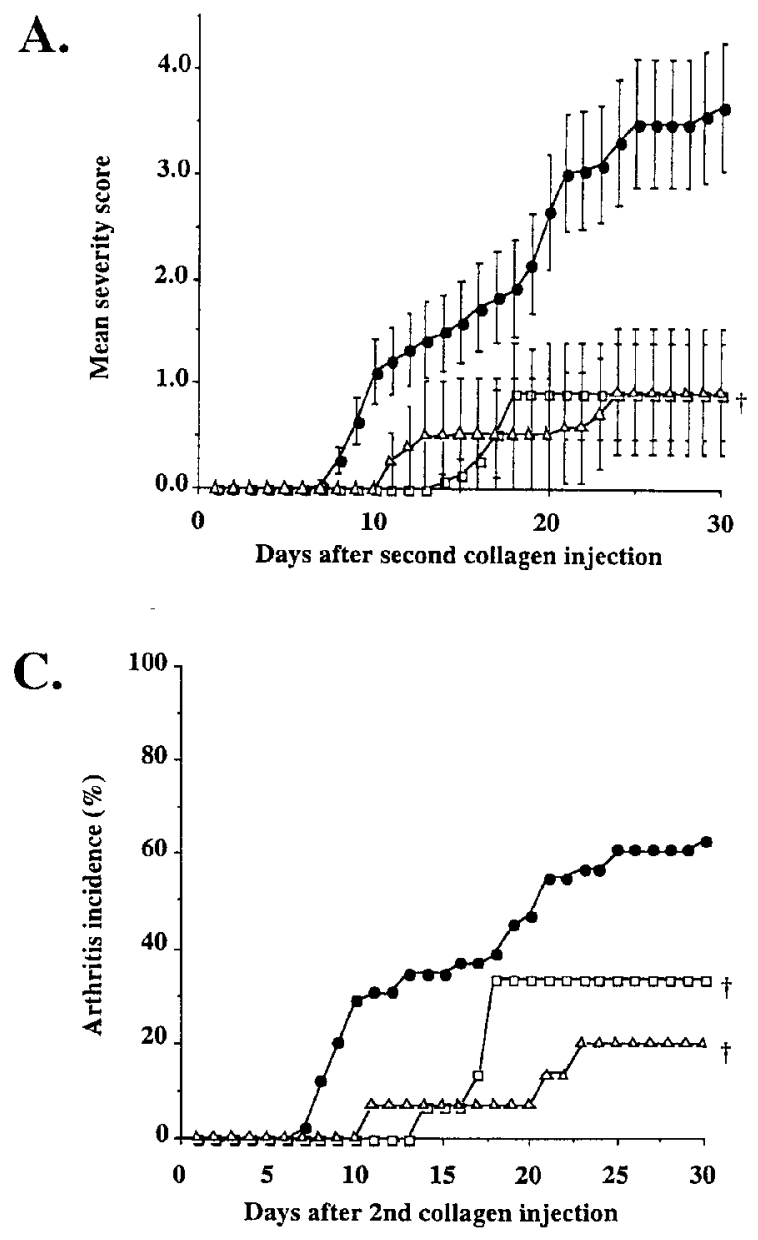

\section{Paw Cytokine Levels}

Paws were snap frozen in liquid nitrogen; the sample was then homogenized in $700 \mu$ l of a TRIS-HCl buffer containing protease inhibitors. Samples were centrifuged for $30 \mathrm{~min}$ and the supernatant frozen at $-80{ }^{\circ} \mathrm{C}$ until assay. Cytokine levels were determined using ELISA (24).

\section{Statistical Analysis}

The results are presented as mean \pm SEM; statistical analysis was preformed using either the Students $t$-test or $\chi^{2}$ test as appropriate, with a $p$ value of less than 0.05 considered significant.

\section{Results}

Arthritis

FP15 treatment at 3 or $10 \mathrm{mg} / \mathrm{kg} / \mathrm{d}$ orally, starting on the day of the second injection of collagen, significantly reduced both the severity (Fig. IA and 1B) and incidence (Fig. 1C) of arthritis in DBA/2J mice. Delaying the start of the FP15 treatment to day 7 after the second collagen injection was also able to reduce the severity of arthritis (Fig. 2A and 2B). However, only the higher dose of FP15 $(10 \mathrm{mg} / \mathrm{kg} / \mathrm{d})$ was able to significantly reduce the
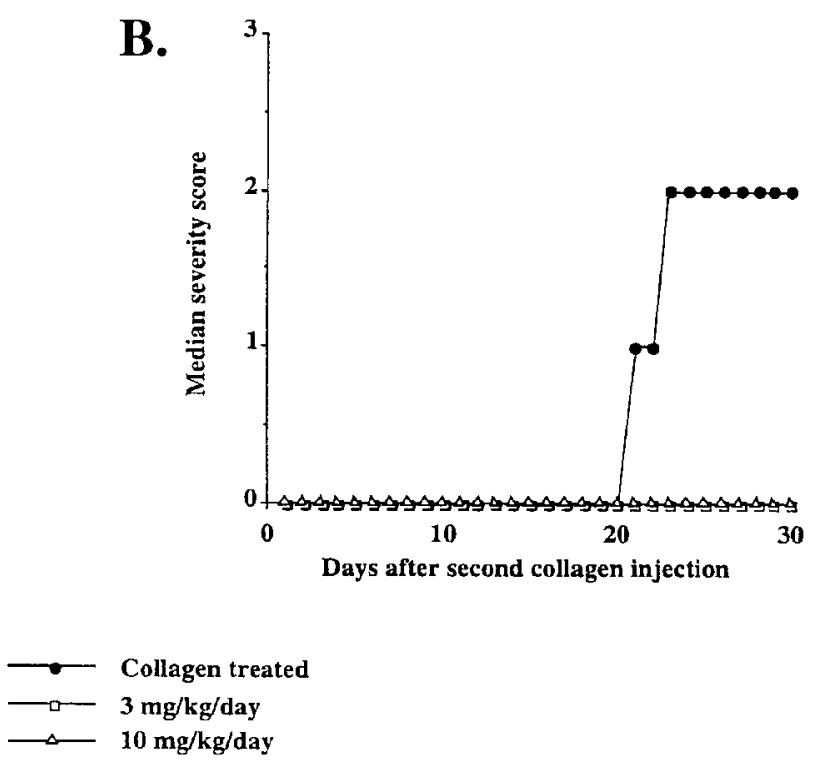

Fig. 1. Effect of FP15 (3 or $10 \mathrm{mg} / \mathrm{kg} / \mathrm{d}$ ) on the severity (A and $B$ ) and incidence (C) of arthritis in DBA/1 J mice. Treatment with FP15 started on the day of the second intradermal injection of collagen and continued throughout the experiment. Severity was assessed daily and expressed as both mean (A) and median (B). As soon as a mouse had a score, it was considered arthritic and the incidence of arthritis (C) was expressed as a cumulative percentage over the 30-day experiment. Results are expressed as mean \pm SEM from 15 mice per experimental group. Statistical analysis was carried out using Student's $t$-test or $\chi^{2}$ test as appropriate. $+p<0.05$ versus vehicle-treated mice. 
incidence of arthritis in the posttreatment paradigm (Fig. 2C)

FP15 at both doses was able to reduce the oxidative stress, as assessed by paw MDA levels, in arthritis (Fig. 3A and 3B) and the effectiveness of the treatment did not depend on when the treatment was started, with delayed treatment with FP15 having similar effects as that starting simultaneously with the second collagen injection. However, there was a difference in effectiveness of treatment timing in neutrophil infiltration of the joint tissue, as assessed by paw MPO levels. FP15 treatment starting simultaneously with the second collagen injection significantly reduced paw MPO levels at both doses used (Fig. 3B). Neither of the doses of FP15, when started 7 days after the second injection of collagen, had any effect on paw MPO levels (Fig. 3B).

Simultaneous treatment with FP15 also significantly reduced the paw levels of the Thl cytokine IL12 (Fig. 4A) and the chemokines MIP-1 $\alpha$ (Fig. 4B). Again, no dose-dependency was observed in these effects. Delaying the lower dose of FP15 to $3 \mathrm{mg} / \mathrm{kg} / \mathrm{d}$ treatment for 7 days was unable to reduce paw levels of IL-12 (Fig. 4A) and only trended to decrease levels of MIP-1 $\alpha$ (Fig. 4B, $p=0.09$ ), However, delayed treatment with $10 \mathrm{mg} / \mathrm{kg} / \mathrm{d}$ of FP15 reduced the paw levels of both IL-12 and MIP- $1 \alpha$ (Fig. 4A and 4B).

A.

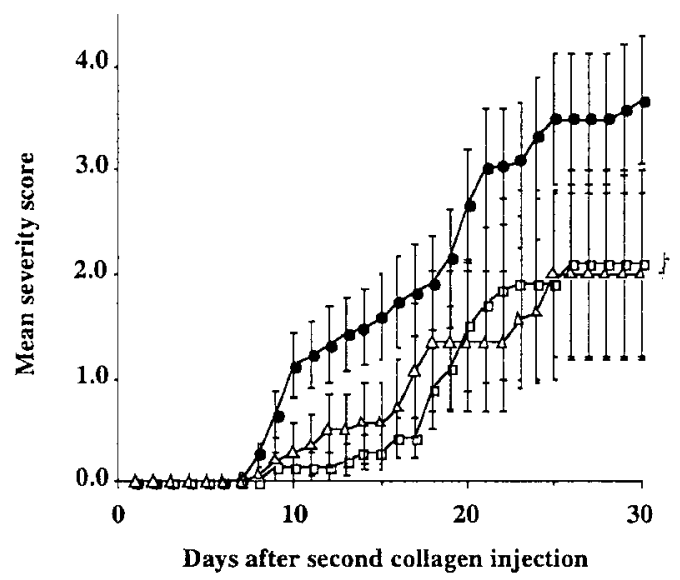

C.

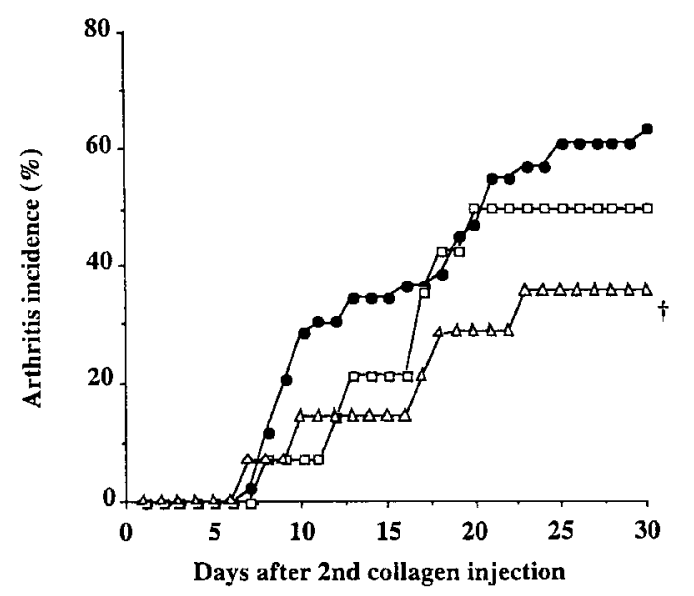

Colitis

FP1 5 treatment $(1,3$, or $10 \mathrm{mg} / \mathrm{kg} / \mathrm{d})$ had no effect on the colitis-induced body weight loss (Fig. 5A). However, the treatment dose-dependently reduced the percentage of mice experiencing rectal bleeding on day 10 after commencing DSS treatment (Fig. 5B). FP15 dose-dependently protected against the DSS-mediated decrease in colon length (Fig. 6A). The increased colon oxidative stress and neutrophil infiltration mediated by colitis were also dose-dependently reduced by FP15 (Fig. 6B and $6 \mathrm{C}$ ), with the lowest dose- $1 \mathrm{mg} / \mathrm{kg} / \mathrm{d}$-having no effect, whereas both 3 and $10 \mathrm{mg} / \mathrm{kg} / \mathrm{d}$ had similar colon protective effects. Colon biopsies taken for histologic analysis were also examined for damage and scored, representative sections for $3 \mathrm{mg} / \mathrm{kg} / \mathrm{d}$ being shown in Figure 7. All FP15 doses used were found to be equally effective in reducing colonic inflammation and mucosal damage (Table 1).

\section{Discussion}

The presented data demonstrate the in vivo effectiveness of FP15, a novel peroxynitrite decomposition catalyst in two animal models of inflammation, arthritis and colitis. FP15 is a novel porphyrin-based peroxynitrite decomposition catalyst that reacts with

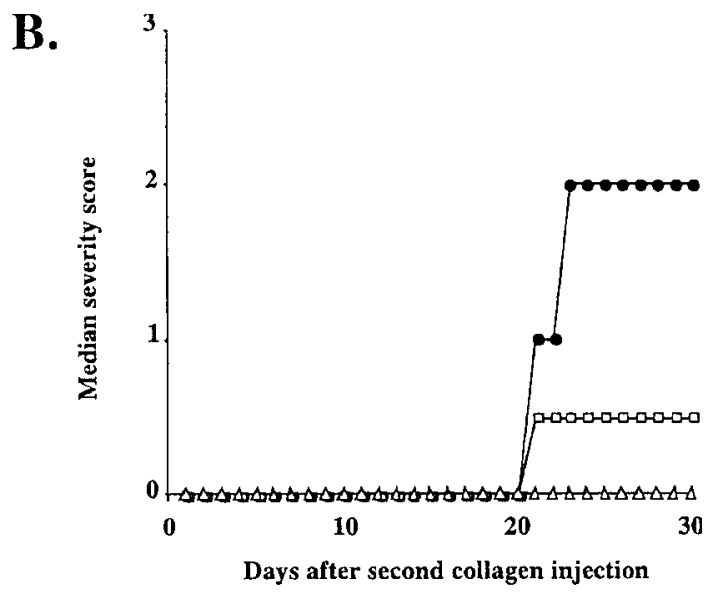

Collagen treated

$3 \mathrm{mg} / \mathrm{kg} / \mathrm{day}$

$10 \mathrm{mg} / \mathrm{kg} / \mathrm{day}$

Fig. 2. Effect of delayed FP15 (3 or $10 \mathrm{mg} / \mathrm{kg} / \mathrm{d}$ ) treatment on the severity (A and $B$ ) and incidence (C) of arthritis in DBA/1J mice. Treatment with FP15 started 7 days after the second intradermal injection of collagen and continued throughout the experiment. Severity was assessed daily and expressed as both mean (A) and median (B). As soon as a mouse had a score, it was considered arthritic and the incidence of arthritis (C) was expressed as a cumulative percentage over the 30-day experiment. Results are expressed as mean \pm SEM from 15 mice per experimental group. Statistical analysis was carried out using Student's $t$-test or $\chi^{2}$ test as appropriate. $+p<0.05$ versus vehicle-treated mice. 


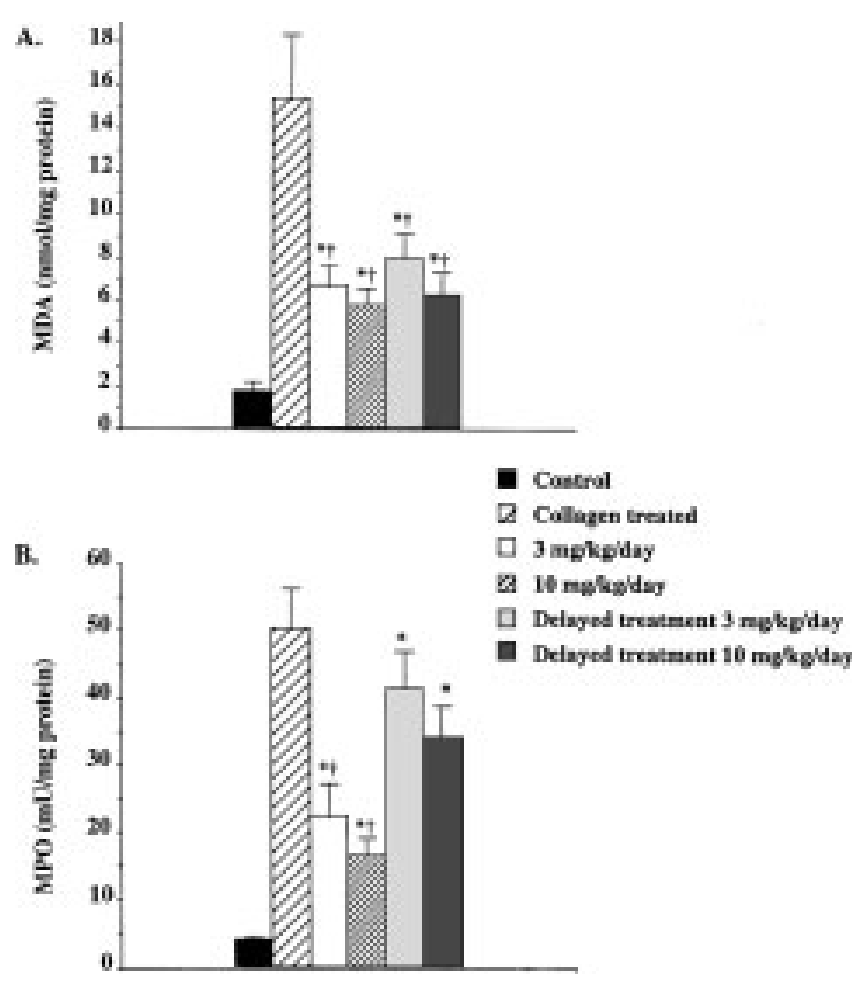

Fig. 3. FP15 ( 3 or $10 \mathrm{mg} / \mathrm{kg} / \mathrm{d})$ treatment effects on the level of (A) MDA and (B) MPO in the paws of mice with arthritis inflammation induced by collagen. Simultaneous treatment with FP15 significantly reduced both MDA and MPO levels in the paws. Delaying FP15 treatment 7 days after the second collagen injection significantly reduced MDA levels, but had no effect on paw MPO levels. Results are expressed as mean \pm SEM from 10 animals. Statistical analysis was conducted using Student's unpaired $t$-test where $p<$ 0.05 was considered significant. ${ }^{*} p<0.05$ versus untreated animals. $+p<0.05$ versus DSS-treated animals.

peroxynitrite with a high rate constant (21). It has recently been shown to be effective in vitro and in vivo, protecting against diabetes mellitus-related endothelial and cardiac dysfunction (21), myocardial ischemia-reperfusion injury (25), pulmonary ischemia- reperfusion injury (26), and doxorubicinmediated myocardial dysfunction (27), conditions in which enhanced superoxide and NO generation, and tyrosine nitration have been documented previously (16). The current extension of the protective effects of FP15 into another two animal models of inflammation is consistent with the hypothesis that (1) peroxynitrite is an important endogenous cytotoxin, which has relevance for the pathogenesis of a variety of cardiovascular and inflammatory diseases and (2) metalloporphyrinic peroxynitrite decomposition catalysts may be effective in treating various inflammatory conditions.

\section{Peroxynitrite and Arthritis}

Prior evidence for the potential pathogenetic role of peroxynitrite in rheumatoid arthritis derives from human studies as well as from experimental data. As
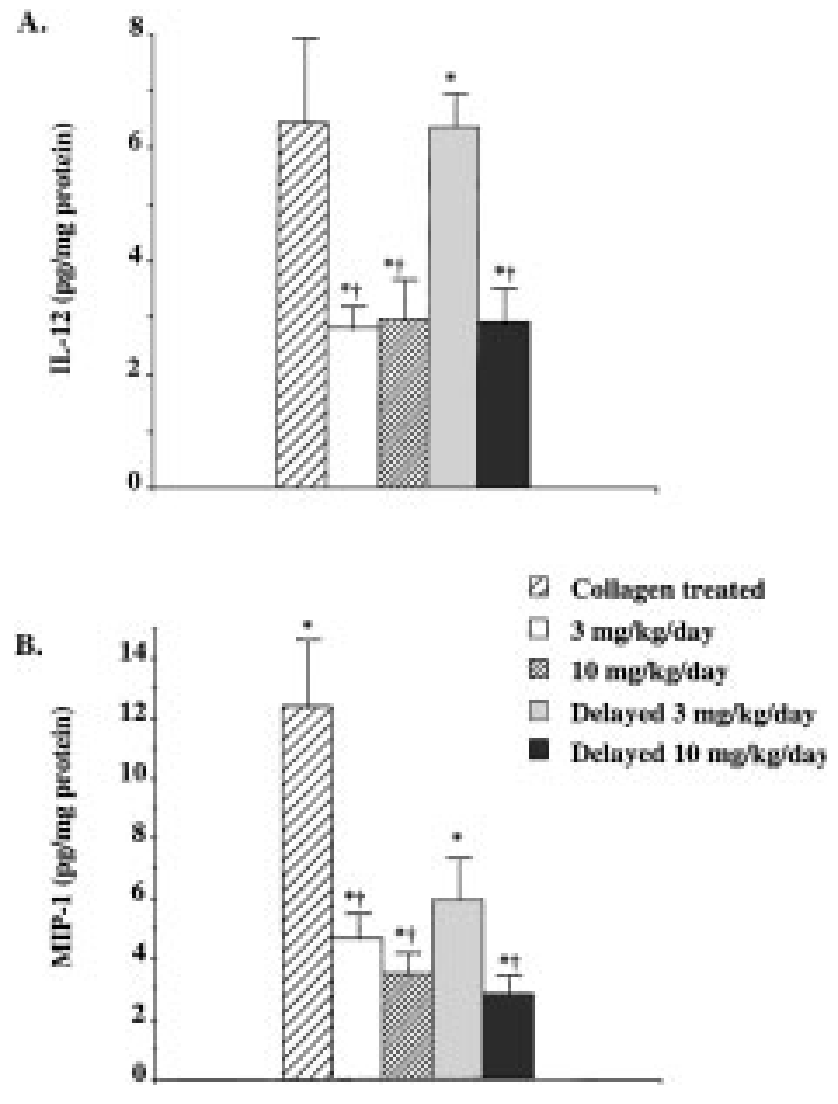

Fig. 4. FP15 (3 or $10 \mathrm{mg} / \mathrm{kg} / \mathrm{d})$ treatment effects on the level of (A) IL-12 and (B) MIP-1 $\alpha$ in the paws of mice with arthritis inflammation induced by collagen. Simultaneous treatment with FP15 significantly reduced both IL-12 and MIP$1 \alpha$ levels in the paws. Delaying FP15 treatment 7 days after the second collagen injection had less of an effect on paw cytokine and chemokines levels, with only the higher dose $(10 \mathrm{mg} / \mathrm{kg} / \mathrm{d})$ significantly reducing both IL-12 and MIP- $1 \alpha$ levels; the lower dose had no significant effect. Results are expressed as mean \pm SEM from 10 animals. Statistical analysis was conducted using Student's unpaired $t$-test where $p<0.05$ was considered significant. ${ }^{*} p<0.05$ versus untreated animals. $+p<0.05$ versus DSS-treated animals.

early as 1994, Kaur and Halliwell (28) demonstrated increased levels of nitrotyrosine with rheumatoid arthritis. Later studies localized nitrotyrosine to the macrophages and the vascular smooth muscle in the inflamed synovium from patients with rheumatoid arthritis (29). Although nitrotyrosine is not fully specific for peroxynitrite formation in vivo-MPO and eosinophil peroxidase-mediated reactions can also yield tyrosine nitration-peroxynitrite appears to be responsible for at least part of its formation in inflammatory conditions $(16,30)$. Our group has previously demonstrated enhanced tyrosine nitration in the inflamed joint extracts in the collagen-induced arthritis model employed in the current study (19). The proposition that peroxynitrite plays a pathogenetic role in arthritis is mainly based on correlative evidence, that is, that (1) NO and superoxide are simultaneously produced in arthritis; (2) the two 

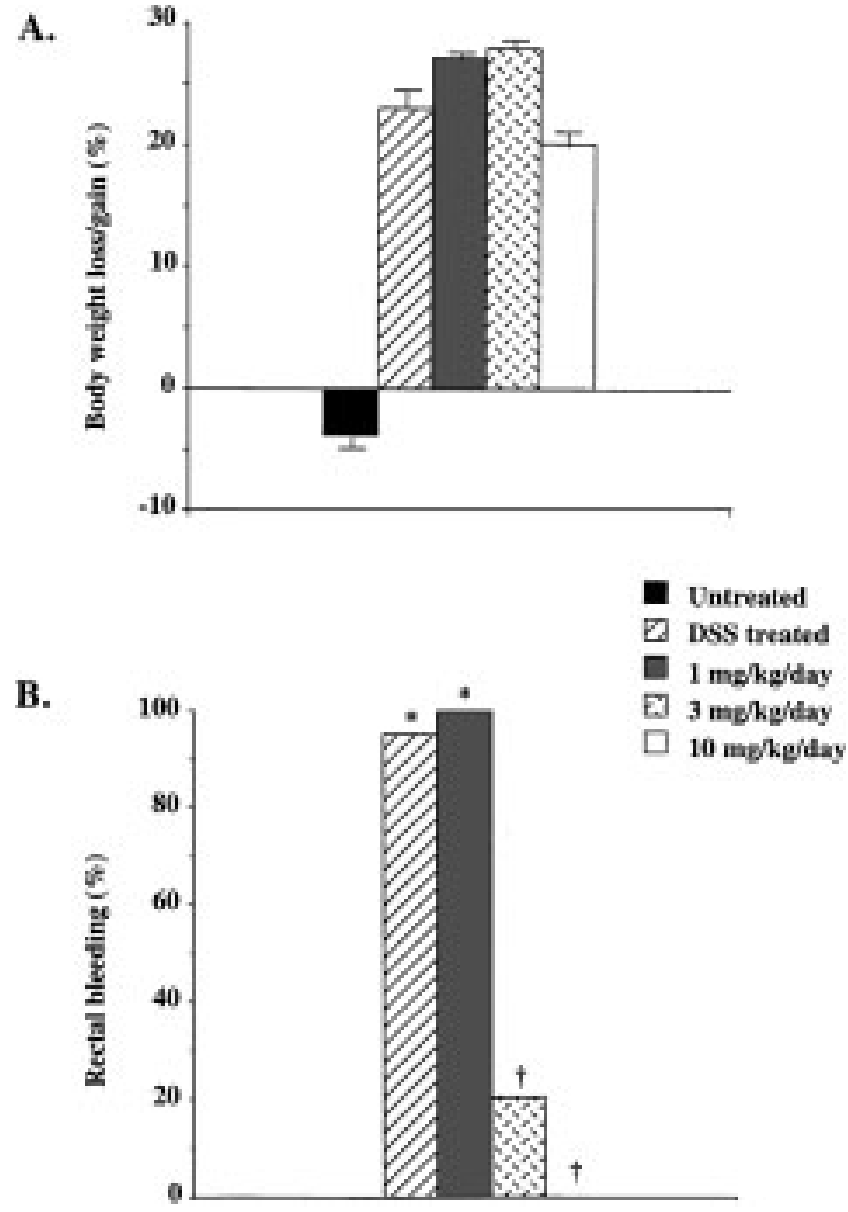

Fig. 5. Effect of FP15 on colitis-induced decreases in (A) body weight and (B) incidence of rectal bleeding. FP15 had no effect on colitis-mediated decrease in body weight, but dose-dependently reduced the incidence of rectal bleeding. Mice were exposed to DSS ad libitum for 10 days; treatment with FP15 (1, 2, or $10 \mathrm{mg} / \mathrm{kg} / \mathrm{d}$, BID) commenced on Day l. Results are expressed as mean \pm SEM from 8-10 animals. Statistical analysis was conducted using Student's unpaired $t$-test where $p<0.05$ was considered significant. ${ }^{*} p<0.05$ versus untreated animals. $+p<0.05$ versus DSS-treated animals.

species are known to react to form peroxynitrite; (3) peroxynitrite is more cytotoxic than NO or superoxide in vitro, and (3) antioxidants or NOS inhibitors that ameliorate arthritis also suppress the formation of nitrotyrosine in the inflamed joint (7-14,31-35). There is also some evidence, in a model of adjuvantinduced arthritis, that earlier generation porphyrinic peroxynitrite decomposition catalysts 5,10,15,20tetrakis(2,4,6-trimethyl-3, 5-disulfonatophenyl)porphyrinato iron (III) and 5,10,15,20-tetrakis(Nmethyl-4' -pyridyl)-porphyrinato iron (III) reduced paw swelling and lactate dehydrogenase release (35).

According to the current data, following induction of arthritis, oral FP15 pretreatment markedly reduces the severity and incidence of the disease. Delaying the start of FP15 administration until the
A.

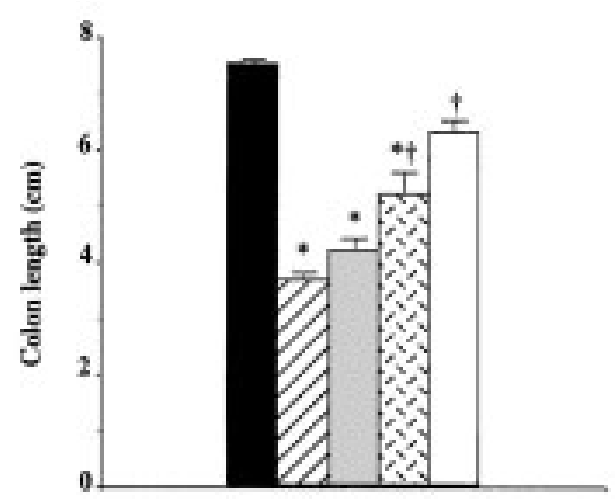

B.

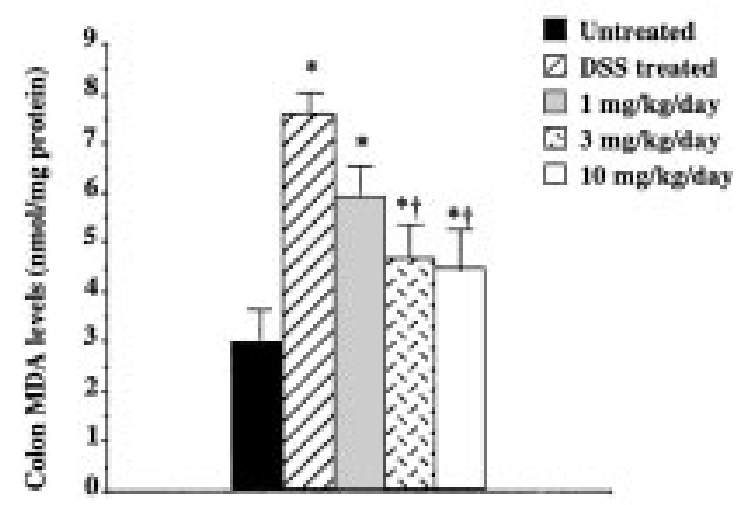

C.

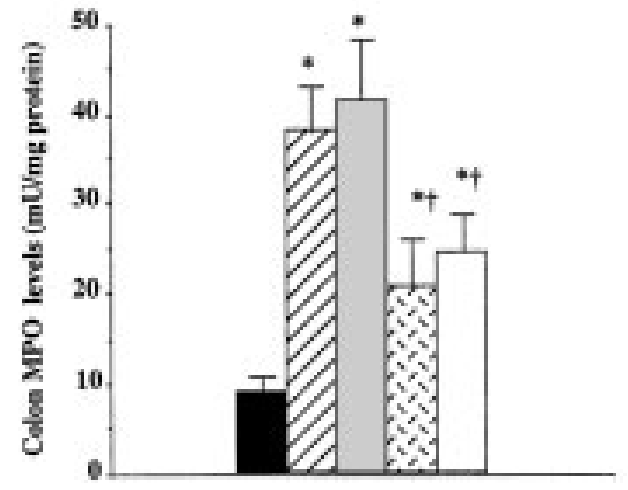

Fig. 6. FP15 dose-dependently increased the (A) colon length and reduced the level of (B) MDA and (C) MPO in the colons of mice with an acute colon inflammation induced by DSS. Mice were exposed to DSS ad libitum for 10 days. Treatment with FP15 (1, 3, or $10 \mathrm{mg} / \mathrm{kg} / \mathrm{d}$, BID) commenced on Day l. Results are expressed as mean \pm SEM from 8-10 animals. Statistical analysis was conducted using Student's unpaired $t$-test where $p<0.05$ was considered significant. ${ }^{*} p<0.05$ versus untreated animals. $+p<0.05$ versus DSS-treated animals.

inflammatory process had begun also attenuated the severity and incidence of arthritis, although not as effectively as when the treatment was started earlier. It is noteworthy that the current experimental model, while amenable to therapeutic efficacy by a variety of agents when applied as pretreatment, is rather difficult to influence in affecting the course of established arthritis, with only a select group of agents, including rapamycin, neutralization of 

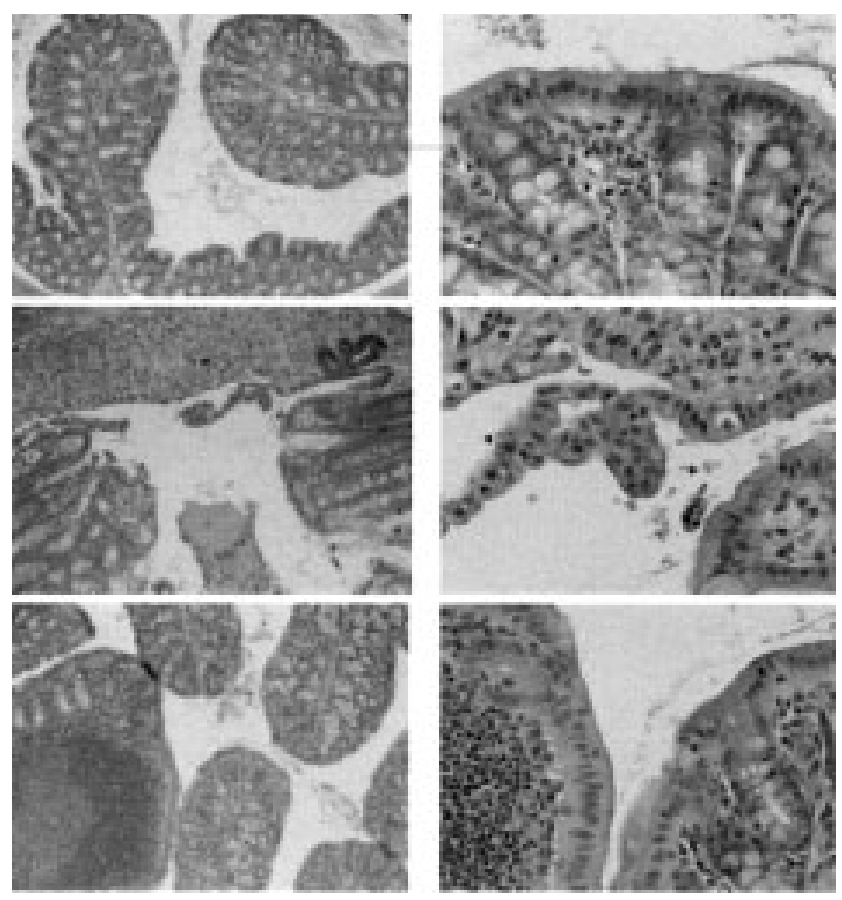

Fig. 7. Effect of FP15 (3 mg/kg/d) on the morphologic changes observed in the colon of mice treated with DSS for 10 days. FP15 was administered orally starting on day 1 . On day 10, mice were killed and colon biopsies taken and fixed in $10 \%$ formalin solution. The samples were embedded in paraffin and sectioned (4- $\mu \mathrm{m}$ sections). The sections were stained with hematoxylin and eosin and viewed at $400 \times$ magnification. (Top). Control. (Middle). DSS. (Bottom). DSS + FP15. (Left panels). Low magnification. (Right panels). High magnification. Sections presented are representative of sections from 10 mice; scoring was carried out by a blinded investigator and reported in Table 1.
VEGF, and IL-10 and IL-4 gene therapy, demonstrating efficacy (36-40). We believe that the reason FP15 maintained some of its efficacy in the delayed mode of administration is due to the fact that NO, superoxide, and peroxynitrite generation represent delayed events in the immunologic and inflammatory series of events associated with arthritis. Indeed, analysis of the paw tissue showed that FP15 effectively reduces the oxidative stress in arthritis, as demonstrated by decreased paw levels of MDA. This effect was observed to a similar extent with both doses of FP15, and was evident both in the pretreatment and in delayed treatment groups. Interestingly, early treatment with FP15 reduced neutrophil infiltration into the paws as indicated by a reduced level of MPO, an effect that was associated with a reduction in paw levels of the chemokine, MIP- $1 \alpha$, and the Thl cytokine IL-12. These effects are most likely brought about by FP15 interrupting the positive feedback cycles of inflammation. In fact, multiple lines of recent evidence indicate that peroxynitrite can promote and enhance pro-inflammatory signal transduction processes (41-44).

\section{Peroxynitrite and Colitis}

Similar to the situation in arthritis, evidence implicating the pathogenetic role of peroxynitrite in the pathogenesis of colitis is mainly based on correlative evidence; that is, that (1) nitrotyrosine has been detected in animal models and human samples of inflammatory bowel disease, (2) NO and superoxide are simultaneously produced in colitis, (3) the two species are known to react to form peroxynitrite, (4) peroxynitrite is more cytotoxic than NO or superoxide in vitro, (5) antioxidants or NOS inhibitors that ameliorate

Table 1. Histologic analysis of colonic sections from, vehicle, DSS + vehicle, and DSS- + FP15-treated mice

\begin{tabular}{lcccc}
\hline Groups & $\begin{array}{c}\text { Inflammation } \\
\text { Severity }\end{array}$ & $\begin{array}{c}\text { Inflammation } \\
\text { Extent }\end{array}$ & $\begin{array}{c}\text { Crypt } \\
\text { Damage }\end{array}$ & Total \\
Vehicle & $0.50 \pm 0.12$ & $0.5 \pm 0.2$ & $0 \pm 0$ & $1.0 \pm 0.4$ \\
DSS + vehicle & $2.5 \pm 0.2$ & $2.5 \pm 0.2$ & $2.2 \pm 0.5$ & $7.2 \pm 0.8$ \\
DSS + FP15 & $1.8 \pm 0.3$ & $1.9 \pm 0.2$ & $0.8 \pm 0.1$ & $4.5 \pm 0.5^{*}$ \\
$(1 \mathrm{mg} / \mathrm{kg} / \mathrm{d})$ & $1.4 \pm 0.2$ & $1.8 \pm 0.1$ & $1.0 \pm 0.4$ & $4.2 \pm 0.7^{*}$ \\
DSS + FP15 & $1.4 \pm 0.2$ & $1.89 \pm 0.10$ & $0.9 \pm 0.3$ & $4.2 \pm 0.6^{*}$
\end{tabular}

Male Balb/c mice were exposed to the DSS solution (5\% w/v) ad libitum. FP15 (1, 3, or $10 \mathrm{mg} / \mathrm{kg} / \mathrm{d})$ was administered orally BID starting on day 1 . On day 10, mice were sacrificed and colon biopsies taken and fixed in $10 \%$ formalin solution. The samples were embedded in paraffin and sectioned (4- $\mu \mathrm{m}$ sections). The section were stained with hematoxylin and eosin, viewed at $400 \times$ magnification, and scored blind for inflammation severity $(0=$ none, $1=$ mild, $2=$ moderate, $3=$ severe $)$ and extent $(0=$ none, $1=$ mucosal, $2=$ mucosal and submucosal, 3 = transmural $)$ as well as crypt damage $(0=$ none, $1=$ basal third, $2=$ basal two-thirds, $3=$ crypts lost epithelium present, $4=$ crypts and surface epithelium lost). The scores are presented as mean \pm SEM from 10 animals. Statistical analysis was conducted using Mann-Whitney test where $*=$ a two-tailed $p$ value less than 0.05 versus the DSS + vehicle treatment group. 
colitis also suppress the formation of nitrotyrosine in the inflamed gut tissue, and (6) application of authentic peroxynitrite to the gut induces a colitis-like inflammatory response $(1-5,12-14,20,45-47)$. Here we report that FP15 dose-dependently attenuated the symptoms of colitis. Histologic analysis of colon biopsies clearly showed the effectiveness of FP15 in reducing colon damage and inflammation. Furthermore, as observed in the arthritis model, FP15 reduced the colitis-induced increase in colonic MDA levels, indicative of the ability of the compound to attenuate oxidative stress. Mice treated with FP15 had significantly longer colons and reduced colon damage and rectal bleeding. As in the arthritis model, FP15 reduced colonic neutrophil infiltration as determined by MPO levels; this effect was only observed at the higher doses. Thus, we believe that the current results are consistent with a causative role of peroxynitrite in the pathogenesis of inflammatory bowel disease.

\section{The Place of Peroxynitrite in the Inflammatory Pathways}

Peroxynitrite appears to play a central role in the pathogenesis of various inflammatory diseases. A proposed scheme of the various interrelated inflammatory pathways upstream and downstream from peroxynitrite is shown in Figure 8. One important advantage of targeting peroxynitrite in inflammatory diseases is related to the distal location of this oxidant in the overall scheme of inflammation, cellular damage, and tissue destruction. One of the major downstream mechanisms by which peroxyni- trite induces cellular damage is via DNA singlestrand breaks and the resulting activation of poly (ADP-ribose) synthetase or polymerase (PARS or PARP) leading to depletion of cellular ATP levels and necrosis (48). PARS inhibitors have been shown to be effective in protecting against both arthritis $(19,49)$ and colitis $(17,50)$. It is likely, therefore, that the neutralization of upstream oxidants such as peroxynitrite will prevent subsequent activation of PARS. This may be one of the protective mechanisms by which FP15 protects against inflammatory tissue damage. In contrast to PARS inhibitors, which affect only a single, albeit important, pathway of inflammation, FP15 is expected to affect a variety of peroxynitrite-mediated cytotoxic pathways; it is expected to prevent DNA injury as well as damage to enzymes involved in cellular energy generation and inflammatory signal transduction $(41-44,49,51)$ and the activation of metalloproteinases $(52,53)$. In a previous study, we demonstrated that the dose of FP15 used in the current study is able to prevent tyrosine nitration (21) and metalloproteinase activation (24) in vivo. Further studies are required to determine to which relative extent the blockade of the above-mentioned various pathways of peroxynitrite toxicity contributes to the beneficial effects reported herein. As discussed previously (21), and despite the extremely high reaction rate constant of FP15 with peroxynitrite (21), it is also conceivable that FP15 (similarly to other porphyrinic antioxidants), exerts additional antioxidant

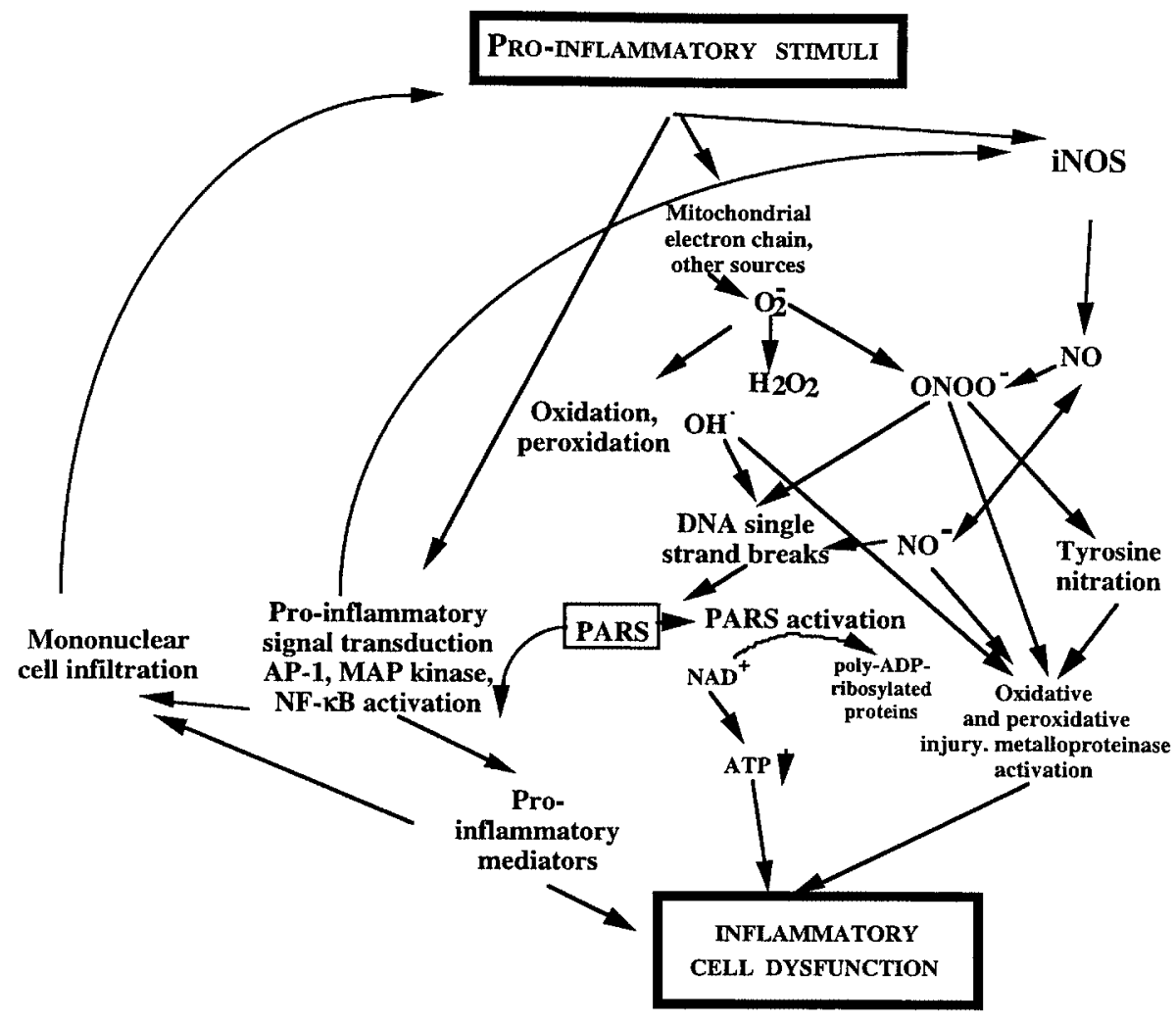

Fig. 8. Proposed scheme of distal inflammatory pathways of arthritis and colitis initiated by free radicals and oxidants. Pro-inflammatory stimuli induce the generation of inflammatory cytokines and chemokines, which in turn trigger various self-amplifying cascades culminating in the generation of oxidants and free radicals, including the expression of iNOS, which in turn produces NO and nitroxyl anion. There is also increased oxidative stress. NO and superoxide react to form peroxynitrite. Peroxynitrite, hydroxyl radical, and nitroxyl anion induce DNA single-strand breakage, as well as increased oxidative stress (including MDA formation). DNA single-strand breakage activates PARS, which in turn further enhances the induction of iNOS, promotes the infiltration of inflammatory mononuclear cells (neutrophils can be quantified by measurement of MPO), can directly trigger necrotic type cell death, and may enhance various pro-inflammatory signal transduction pathways. 
effects on other free radical systems such as superoxide $(34,54)$.

Overall, the results presented here support the notion that FP15 or other porphyrinic antioxidant molecules should be further explored for the experimental therapy of human inflammatory diseases.

\section{Acknowledgments}

This work was supported by a grant from the National Institutes of Health (R44DK53675).

\section{References}

1. Singer II, Kawka DW, Scott S, et al. (1996) Expression of inducible nitric oxide synthase and nitrotyrosine in colonic epithelium in inflammatory bowel disease. Gastroenterology 111: 871-885.

2. Zingarelli B, Cuzzocrea S, Szabo C, Salzman AL. (1998) Mercaptoethylguanidine, a combined inhibitor of nitric oxide synthase and peroxynitrite scavenger, reduces trinitrobenzene sulfonic acid- induced colonic damage in rats. J. Pharmacol. Exp. Ther. 287: 1048-1055.

3. Cuzzocrea S, Mazzon E, Dugo L, Caputi AP, Riley DP, Salvemini D. (2001) Protective effects of M40403, a superoxide dismutase mimetic, in a rodent model of colitis. Eur. $J$. Pharmacol. 432: 79-89.

4. Goggins MG, Shah SA, Goh J, et al. (2001) Increased urinary nitrite, a marker of nitric oxide, in active inflammatory bowel disease. Mediators Inflamm. 10: 69-73.

5. Perner A, Nordgaard I, Matzen P, Rask-Madsen J. (2002) Colonic production of nitric oxide gas in ulcerative colitis, collagenous colitis and uninflamed bowel. Scand. J. Gastroenterol. 37: 183-188.

6. Evans CH, Stefanovic-Racic M, Lancaster J. (1995) Nitric oxide and its role in orthopaedic disease Clin. Orthop. 312: 275-294.

7. Santos LL, Morand EF, Yang Y, Hutchinson P, Holdsworth SR. (1997) Suppression of adjuvant arthritis and synovial macrophage inducible nitric oxide by $\mathrm{N}$-iminoethyl-L-ornithine, a nitric oxide synthase inhibitor. Inflammation 21: 299-311.

8. Brahn E, Banquerigo ML, Firestein GS, Boyle DL, Salzman AL, Szabo C. (1998) Collagen induced arthritis: reversal by mercaptoethylguanidine, a novel antiinflammatory agent with a combined mechanism of action. J. Rheumatol. 25: 1785-1793.

9. Salvemini D, Mazzon E, Dugo L, et al. (2001) Amelioration of joint disease in a rat model of collagen-induced arthritis by M40403, a superoxide dismutase mimetic. Arthritis Rheum. 44: 2909-2921.

10. Attur MG, Patel RN, Abramson SB, Amin AR. (1997) Interleukin-17 up-regulation of nitric oxide production in human osteoarthritis cartilage. Arthritis Rheum. 40: 1050-1053.

11. Stichtenoth DO, Fauler J, Zeidler H, Frolich JC. (1995) Urinary nitrate excretion is increased in patients with rheumatoid arthritis and reduced by prednisolone. Ann. Rheum. Dis. 54: 820-824.

12. Koppenol WH, Moreno JJ, Pryor WA, Ischiropoulos H, Beckman JS. (1992) Peroxynitrite, a cloaked oxidant formed by nitric oxide and superoxide. Chem. Res. Toxicol. 5: 834842.

13. Hausladen A, Fridovich I. (1994) Superoxide and peroxynitrite inactivate aconitases, but nitric oxide does not. J. Biol. Chem. 269: 29405-29408.

14. Szabo C, Day BJ, Salzman AL. (1996) Evaluation of the relative contribution of nitric oxide and peroxynitrite to the suppression of mitochondrial respiration in immunostimulated macrophages using a manganese mesoporphyrin superoxide dismutase mimetic and peroxynitrite scavenger. FEBS Lett. 381: 82-86.

15. Szabo C, Zingarelli B, O'Connor M, Salzman AL. (1996) DNA strand breakage, activation of poly (ADP-ribose) synthetase, and cellular energy depletion are involved in the cytotoxicity of macrophages and smooth muscle cells exposed to peroxynitrite. Proc. Natl. Acad. Sci. U.S.A. 93: 1753-1758.

16. Greenacre SA, Ischiropoulos H. (2001) Tyrosine nitration: localisation, quantification, consequences for protein function and signal transduction. Free Radic. Res. 34: 541-581.

17. Zingarelli B, Szabo C, Salzman AL. (1999) Blockade of Poly(ADP-ribose) synthetase inhibits neutrophil recruitment, oxidant generation, and mucosal injury in murine colitis. Gastroenterology 116: 335-345.

18. Szabo C, Scott GS, Virag L, et al. (1998) Suppression of macrophage inflammatory protein (MIP)-lalpha production and collagen-induced arthritis by adenosine receptor agonists. Br. J. Pharmacol. 125: 379-387.

19. Szabo C, Virag L, Cuzzocrea S, et al. (1998) Protection against peroxynitrite-induced fibroblast injury and arthritis development by inhibition of poly(ADP-ribose) synthase. Proc. Natl. Acad. Sci. U.S.A. 95: 3867-3872.

20. Rachmilewitz D, Stamler JS, Karmeli F, et al. (1993) Peroxynitrite-induced rat colitis-a new model of colonic inflammation. Gastroenterology 105: 1681-1688.

21. Szabó C, Mabley JG, Moeller SM, et al. (2002) Pathogenetic role of peroxynitrite in the development of diabetes and diabetic vascular complications: studies with FP15, a novel potent peroxynitrite decomposition catalyst. Mol. Med. 8(10): 571-580.

22. Liaudet L, Mabley JG, Pacher P, et al. (2002) Inosine exerts a broad range of antiinflammatory effects in a murine model of acute lung injury. Ann. Surg. 235: 568-578.

23. Bradford MM. (1976) A rapid and sensitive method for the quantification of microgram quantities of protein utilizing the principle of protein-dye binding. Anal. Biochem. 72: 248-254.

24. Mabley JG, Pacher P, Southan GJ, Salzman AL, Szabo C. (2002) Nicotine reduces the incidence of type I diabetes in mice. J. Pharmacol. Exp. Ther. 300: 876-881.

25. Bianchi C, Wakiyama H, Faro R, et al. (2002) A novel peroxynitrite decomposition catalyst (FP-15) reduces heart infarct size in a model of acute ischemia-reperfusion. Ann. Thorac. Surg. (in press).

26. Pacher P, Liaudet L, Bai P, Virág L, Mabley JG, Szabó C. (2002) A potent peroxynitrite decomposition catalyst, FP15, protects against the development of doxorubicin-induced heart failure. FASEB J. 16: A177 (abstract).

27. Naidu BV, Krishnadasan B, Fraga C, et al. (2002) The critical role of reactive nitrogen species in lung ischemia reperfusion injury. J. Heart Lung Transplant. 21 : 135 (abstract).

28. Kaur H, Halliwell B. (1994) Evidence for nitric oxide-mediated oxidative damage in chronic inflammation. Nitrotyrosine in serum and synovial fluid from rheumatoid patients. FEBS Lett. 350: 9-12.

29. Mapp PI, Klocke R, Walsh DA, et al. (2001) Localization of 3nitrotyrosine to rheumatoid and normal synovium. Arthritis Rheum. 44: 1534-1539.

30. Brennan ML, Wu W, Fu X, et al. (2002) A tale of two controversies: defining both the role of peroxidases in nitrotyrosine formation in vivo using eosinophil peroxidase and myeloperoxidase-deficient mice, and the nature of peroxidasegenerated reactive nitrogen species. J. Biol. Chem. 277: 1741517427.

31. Salvemini D, Wang ZQ, Bourdon DM, Stern MK, Currie MG, Manning PT. (1996) Evidence of peroxynitrite involvement in the carrageenan-induced rat paw edema. Eur. J. Pharmacol. 303: 217-220.

32. Pelletier JP, Lascau-Coman V, Jovanovic D, et al. (1999) Selective inhibition of inducible nitric oxide synthase in experimental osteoarthritis is associated with reduction in tissue levels of catabolic factors. J. Rheumatol. 26: 2002-2014.

33. Keng T, Privalle CT, Gilkeson GS, Weinberg JB. (2000) Peroxynitrite formation and decreased catalase activity in autoimmune MRL-lpr/lpr mice. Mol. Med. 6: 779-792.

34. Del Carlo M Jr, Loeser RF. (2002) Nitric oxide-mediated chondrocyte cell death requires the generation of additional reactive oxygen species. Arthritis Rheum. 46: 394-403. 
35. Salvemini D, Wang ZQ, Stern MK, Currie MG, Misko TP. (1998) Peroxynitrite decomposition catalysts: therapeutics for peroxynitrite-mediated pathology. Proc. Natl. Acad. Sci. U.S.A. 95: 2659-2663.

36. Carlson RP, Hartman DA, Tomchek LA, et al. (1993) Rapamycin, a potential disease-modifying antiarthritic drug. $J$. Pharmacol. Exp. Ther. 266: 1125-1138.

37. Williams PJ, Jones RH, Rademacher TW. (1997) Reduction in the incidence and severity of collagen-induced arthritis in DBA/1 mice, using exogenous dehydroepiandrosterone. Arthritis Rheum. 40: 907-911.

38. Quattrocchi E, Dallman MJ, Dhillon AP, Quaglia A, Bagnato G, Feldmann M. (2001) Murine IL-10 gene transfer inhibits established collagen-induced arthritis and reduces adenovirus-mediated inflammatory responses in mouse liver. $J$. Immunol. 166: 5970-5978.

39. Kim SH, Kim S, Evans CH, Ghivizzani SC, Oligino T, Robbins PD. (2001) Effective treatment of established murine collagen-induced arthritis by systemic administration of dendritic cells genetically modified to express IL-4. J. Immunol. 166: 3499-3505.

40. Sone H, Kawakami Y, Sakauchi M, et al. (2001) Neutralization of vascular endothelial growth factor prevents collageninduced arthritis and ameliorates established disease in mice. Biochem. Biophys. Res. Commun. 281: 562-568.

41. Gertzberg N, Clements R, Jaspers I, et al. (2000) Tumor necrosis factor-alpha-induced activating protein- 1 activity is modulated by nitric oxide-mediated protein kinase $\mathrm{G}$ activation. Am. J. Respir. Cell. Mol. Biol. 22: 105-115.

42. Cooke CL, Davidge ST. (2002) Peroxynitrite increases iNOS through NF-kappaB and decreases prostacyclin synthase in endothelial cells. Am. J. Physiol. 282: C395-C402.

43. Zouki C, Jozsef L, Ouellet S, Paquette Y, Filep JG. (2001) Peroxynitrite mediates cytokine-induced IL-8 gene expression and production by human leukocytes. J. Leukoc. Biol. 69: 815-824.
44. Matata BM, Galinanes M. (2002) Peroxynitrite is an essential component of cytokines production mechanism in human monocytes through modulation of nuclear factor-kappa B DNA binding activity. J. Biol. Chem. 277: 2330-2335.

45. Singer II, Kawka DW, Scott S, et al. (1996) Expression of inducible nitric oxide synthase and nitrotyrosine in colonic epithelium in inflammatory bowel disease. Gastroenterology 111 : 871-885.

46. Miampamba M, Sharkey KA. (1999) Temporal distribution of neuronal and inducible nitric oxide synthase and nitrotyrosine during colitis in rats. Neurogastroenterol. Motil. 11: 193-206.

47. Dijkstra G, Moshage H, van Dullemen HM, et al. (1998) Expression of nitric oxide synthases and formation of nitrotyrosine and reactive oxygen species in inflammatory bowel disease. J. Pathol. 186: 416-421.

48. Virág L, Szabó C. (2002) The therapeutic potential of PARP inhibition. Pharmacol. Rev. 54: 375-429.

49. Mabley JG, Jagtap P, Perretti M, et al. (2001) Anti-inflammatory effects of a novel, potent inhibitor of poly (ADP-ribose) polymerase. Inflamm. Res. 50: 561-569.

50. Radi R, Cassina A, Hodara R. (2002) Nitric oxide and peroxynitrite interactions with mitochondria. Biol. Chem. 383: 401-409.

51. Szabo C, Ohshima H. (1997) DNA damage induced by peroxynitrite: subsequent biological effects. Nitric Oxide 1: 373385.

52. Okamoto T, Akaike T, Sawa T, Miyamoto $\mathrm{Y}$, van der Vliet A, Maeda H. (2001) Activation of matrix metalloproteinases by peroxynitrite-induced protein S-glutathiolation via disulfide S-oxide formation. J. Biol. Chem. 276: 29596-29602.

53. Wang W, Sawicki G, Schulz R. (2002) Peroxynitrite-induced myocardial injury is mediated through matrix metalloproteinase-2. Cardiovasc. Res. 53: 165-174.

54. Macmillan-Crow LA, Cruthirds DL. (2001) Manganese superoxide dismutase in disease. Free Radic. Res. 34: 325-336. 